\title{
ESCOLARIDAD Y ENTRENAMIENTO EN EL MODELO DE SOLUCIÓN DE PROBLEMAS PARA EL MANEJO DE LA DIABETES MELLITUS TIPO 2
}

\author{
SCHOOLING AND TRAINING IN THE \\ PROBLEM SOLVING MODEL FOR THE \\ MANAGEMENT OF TYPE 2 DIABETES \\ MELLITUS
}

\author{
YOLANDA RODRÍGUEZ SORIANO ${ }^{1}$, JORGE MARTÍNEZ STACK ${ }^{1}$, \\ MA. DE LOURDES RODRÍGUEZ CAMPUZANO ${ }^{1} \mathrm{Y}$ \\ ANTONIO ROSALES ARELLANO ${ }^{1}$
}

Cómo referenciar este artículo/How to reference this article:

Rodríguez Soriano, Y., Martínez Stack, J., Rodríguez Campuzano, M. L. y Rosales Arellano, A. (2020). Escolaridad y entrenamiento en el modelo de Solución de Problemas para el manejo de la Diabetes Mellitus Tipo 2 [Schooling and Training in the Problem Solving Model for the Management of Type 2 Diabetes Mellitus]. Acción Psicológica, 17(2), 57-70. https://doi.org/10.5944/ap.17.2.27955

\section{Resumen}

Mediante un modelo de capacitación en solución de problemas aplicado a una población mexicana con diabetes Melitus2 (DM2), se investigó la influencia del grado de escolaridad en la adquisición de habilidades de solución de problemas para el control de la glucemia. Participaron 20 adultos diagnosticados con DM2, 12 hombres y ocho mujeres, con una edad promedio de 60.17 años los hombres y 61.25 años las mujeres y con una escolaridad media de 15.67 y 9.13 años de los hombres y mujeres respectivamente. Antes y después del taller de capacitación presencial, se estimó el grado de habilidades en solución de problemas mediante un cuestionario de 16 reactivos, así como el grado de glucemia. La comparación entre las mediciones de antes y después del taller mostró que el grupo de mayor escolaridad obtuvo mejores calificaciones que el de menor escolaridad, tanto en la prueba de solución de problemas como en el control de la

Correspondence address [Dirección para correspondencia]: Norma Yolanda Rodríguez Soriano, Facultad de Estudios Superiores, Universidad Nacional Autónoma de México, México.

Email: normayolanda@gmail.com

ORCID: Norma Yolanda Rodríguez Soriano (http://orcid.org/0000-0003-0553-6659).

${ }^{1}$ Universidad Autónoma de México, México.

Recibido: 3 de agosto de 2020.

Aceptado: 13 de diciembre de 2020. 
glucemia. El resultado confirma la importancia de considerar en el diseño instruccional de talleres de capacitación en habilidades de solución de problemas, el nivel educativo de la población a la que van dirigidos, con el propósito de mejorar las herramientas disponibles para el manejo de la glucemia.

Palabras clave: solución de problemas; glucemia; DM2; escolaridad.

\begin{abstract}
Using a problem-solving training model applied to a Mexican population sample with diabetes mellitus2 (DM2), the influence of the level of schooling in the acquisition of problem-solving skills for glycemic control was investigated. Twenty adults diagnosed with DM2 participated, 12 men and 8 women, with an average age of 60.17 years for men and 61.25 years for women and with an average schooling of 15.67 and 9.13 years for men and women, respectively. Before and after the faceto-face training workshop, the degree of problem-solving skills was estimated using a 16 -item questionnaire, as well as the level of blood glucose. The comparison between the measurements before and after the workshop showed that the group with the highest level of education obtained better grades than the one with the lowest level of education, both in the problem-solving test and in the glycemic control. The result confirms the importance of considering in the instructional design of training workshops in problem-solving skills, the educational level of the target population, in order to improve the tools available for glycemic management.
\end{abstract}

Keywords: problem solving; blood glucose; DM2; schooling.

\section{Introducción}

La Organización Mundial de la Salud (OMS, 2020a) define a la Diabetes Mellitus (DM) como una enfermedad crónica que afecta sistémicamente y se desarrolla cuando el organismo no utiliza eficientemente una hormona que produce el páncreas (insulina) y que es la encargada de regular la glucosa en sangre (DM1); o cuando el páncreas no la produce suficientemente (DM2). Conforme a estimaciones de las propia OMS, la amenaza se incrementa constantemente porque el número de adultos con DM pasó de 108 millones en 1980, a 422 millones en 2014. Cada año, la DM causa alrededor de 1.5 millones de muertes en el mundo, además de otros 2.2 millones de defunciones que son atribuidas a alteraciones fisiológicas ocasionadas por la hiperglucemia, lo que supone un total anual de $3.7 \mathrm{mi}-$ llones de muertes vinculadas con la permanencia de altos niveles de glucosa en sangre (Chan, 2016).

En México, se reporta que la DM ocasiona el 14\% del total de muertes en todas las edades, además de que su prevalencia exhibe una curva de crecimiento sostenido desde 1980 (OMS, 2020b). La Encuesta Nacional de Salud (Instituto Nacional de Salud Pública de México, 2018), informó que hay 8.6 millones de mexicanos que viven con diabetes, $11.4 \%$ son mujeres y $09.1 \%$ son hombres. En ambos casos, las cifras incrementaron de 2012 a 2018.

Un primer elemento de prevención de DM es su diagnóstico temprano, con el propósito de que el paciente se familiarice con las características de la enfermedad para que la integre como una condición personal y actúe en consecuencia, ya que enfrentar el padecimiento requiere de generar un cambio en el estilo de vida (García y Sánchez, 2013).

La Asociación Americana de Educadores en Diabetes (AADE, 2018 por sus siglas en inglés), propone un esquema de siete pasos para que el paciente adquiera los hábitos que le ayudarán a superar las eventuales barreras en el cuidado de su salud, a prevenir complicaciones asociadas y a mejorar su calidad de vida. Estos pasos son: ingerir comida saludable, estar activo, monitorear la glucemia, tomar el medicamento, resolver problemas, reducir el riesgo de complicaciones y contar con una forma de afrontamiento definida como saludable. La propuesta es semejante a la de García y Sánchez (2013) quienes mencionan que el tratamiento de la DM requiere de la participación del paciente en términos de automonitoreo continuo, adherencia al régimen alimenticio, realización de ejercicio y, 
si fuera el caso, administración adecuada del medicamento.

De acuerdo con Powers et al. (2017), existen al menos cuatro momentos clave, en los que el equipo de salud debe acompañar al paciente durante el proceso de aprender a vivir con DM: al recibir el diagnóstico, durante las evaluaciones de prevención de complicaciones y revisión de necesidades, cuando se presenten nuevas complicaciones que impacten en el autocuidado y, por último, cuando haya cambios en la vida rutinaria que exijan un nuevo ajuste en el manejo de la DM. En este contexto han propuesto el protocolo "Diabetes Self-Management Educational and Support" (DSME/S por sus siglas inglés), para el manejo de la DM en la vida diaria.

En síntesis, Powers et al. (2017) y la AADE (2018) determinan que el cuidado efectivo de la DM requiere de competencias para auto evaluar las características personales, analizar situaciones, identificar opciones, planear acciones y poner en práctica las opciones planeadas; una serie de habilidades compatibles con el modelo de Solución de Problemas (SP), desarrollado por D'Zurilla y Golfried (1971) y revisado después por D'Zurilla y Nezu (2010). A más de treinta años de la propuesta original, Nezu et al. (2015) confirman que la SP es una estrategia vigente para tratar a personas con enfermedades crónicas, tal como lo es la DM.

Recibir el diagnóstico de una enfermedad crónica y degenerativa como la $\mathrm{DM}$, es una situación problemática que requiere de habilidades específicas para aprender a vivir con ella y seguir las indicaciones del médico tratante (Interial et al., 2013; Lazcano y Salazar, 2007; Riveros et al., 2005). Para desarrollar estas habilidades, la técnica de SP ha sido una opción viable y promisoria por sus resultados (Hill-Brigs y Gremmell, 2007; Nezu et al., 2015; Powers et al., 2017; Schumann et al., 2011).

Hill-Briggs (2003) fue uno de los autores que por primera vez reportó la aplicación de esta técnica en el ámbito de la salud, y propuso el modelo de SP en el autocuidado de la DM con base en cuatro componentes: Habilidades en SP, Orientación hacia la SP, Conocimiento específico de la enfermedad y Transferencia de experiencias pasadas. La autora parte del principio de que las enfermedades cróni- cas como la DM son auto manejables, es decir, el paciente es quien monitorea diariamente su estado de salud y toma decisiones con respecto de las opciones disponibles en su entorno. Puesto que el fortalecimiento de esta habilidad de automanejo es hacia donde se debe dirigir la intervención y la capacitación de los pacientes, se toma como base el constructo de SP para ilustrar el proceso de auto manejo de la enfermedad y, a la técnica de SP, como procedimiento para superar las barreras que diariamente se enfrentan al intentar la adherencia a las prescripciones médicas.

Posteriormente, Hill-Briggs y Gemmell (2007) llevaron a cabo un meta-análisis de trabajos publicados entre 1990 y 2006 en los que se alude a la SP como estrategia para mejorar el control y automanejo de la DM. De los 52 estudios analizados, solamente ocho son de intervención en adultos y los efectos reportados hacen referencia a conductas de automanejo, hábitos alimenticios, automonitoreo de glucosa, ejercicio, adherencia al tratamiento médico, decremento en niveles de hemoglobina glucosilada y decremento de peso.

Sin duda, la técnica de SP se sigue utilizando como estrategia de intervención en pacientes diagnosticados con DM. Schumann et al. (2011), publicaron que la técnica es un recurso esencial en el automanejo efectivo de la DM, debido a los resultados que se obtienen en el mejoramiento del estado de salud y el control de la enfermedad. Incluso, consideran que la técnica per se, es suficiente para el automanejo de la DM y en su proyecto DECIDE (Decisionmaking Education for Choices In Diabetes Everyday), pusieron a prueba esta estrategia, encontrando que una de sus principales repercusiones fue que los cuidadores dejaron de asumir como propio el trabajo que competía a los pacientes, quienes comenzaron a colaborar tanto en el cuidado de su propia salud, como en el análisis de prioridades entre los muchos obstáculos que representa el manejo efectivo de la enfermedad. Después del tratamiento, los participantes mejoraron en su conocimiento de la DM, SP y autocuidado; también redujeron el nivel de hemoglobina glucosilada, presión arterial y colesterol LDL. El trabajo muestra la importancia de involucrar activamente a los pacientes en el cuidado de su salud. 
Recientemente, Zhang et al. (2018) realizaron un estudio para entender hasta qué punto los pacientes con DM que participan en comunidades virtuales necesitan información para tomar decisiones en relación con la enfermedad. Analizaron 801 comentarios en línea ("posts") provenientes de una comunidad de DM y encontraron que la necesidad de información se relaciona con los cambios corporales asociados a la enfermedad, el incremento en el auto monitoreo y el conflicto cognitivo que puede generarse al contrastar las creencias personales con la información que se recibe. El trabajo pone de manifiesto, por un lado, la inquietud de los pacientes por las características de la enfermedad y, por otro, la discrepancia entre los intereses del equipo médico y del paciente con DM. Mientras el equipo médico tiene la ruta perfectamente trazada y establece las indicaciones para el manejo óptimo de la enfermedad, las personas con DM están preocupadas por conocer los efectos de la enfermedad e incorporar el diagnóstico a su propia estructura cognitiva, investigando los síntomas así como justificando su nuevo estilo de vida, y fuentes que lo validen; esta discrepancia de intereses médico-paciente termina generando un conflicto y obstaculiza la toma de decisiones efectivas y necesarias para el automanejo de la DM.

Es evidente que el desarrollo de las habilidades para la solución de problemas demanda algo más que la propuesta de un programa, que, si bien ha demostrado efectividad para auto manejar efectivamente la DM2, también requiere de una serie de precurrentes que hacen necesario el ajuste de los contenidos, actividades y lenguaje del programa. Ya lo han mencionado antes, autores como De Vicenzi y Tudesco (2003), así como la Organización de las Naciones Unidas para la Educación la Ciencia y la Cultura (UNESCO, 2020) cuando sugieren que la educación, estimada en términos de años de escolaridad, es un elemento básico para considerar en las propuestas de políticas públicas dirigidas a promover el cuidado de la salud, tanto en el nivel individual como en el comunitario.

Las implicaciones de esta postura se relacionan con el diseño, programación y duración de los cursos de capacitación en el área de la salud; además con la evaluación variada y efectiva de los asistentes o poblaciones a los que se dirigen los cursos. Todos estos aspectos atañen directamente a diseñadores y desarrolladores de iniciativas edu- cativas formales para la promoción de la salud (Sotaminga, 2019).

Tomando como base la capacitación de personas diagnosticadas con DM2 en un modelo de SP para enfrentar el problema de la glucemia, el presente estudio se propuso como objetivo determinar el grado en el que la escolaridad pudiera determinar la eficacia de la capacitación.

El trabajo fue dirigido por las siguientes hipótesis:

$\mathrm{H}_{1}$ : Las diferencias entre el antes y después del curso de capacitación en las calificaciones en solución de problemas serán mayores entre el grupo de mayor escolaridad, en comparación con el grupo de menor escolaridad.

$\mathrm{H}_{2}$ : En el grupo de mayor escolaridad, la diferencia en las mediciones de glucemia, antes y después del curso de capacitación en SP, será mayor a la observada en el grupo de menor escolaridad 1.

\section{Método}

\section{Participantes}

La muestra fue no probabilística por conveniencia (Arias et al., 2016), compuesta por 20 adultos, 12 hombres con una edad media de 60.17 años (con un rango entre 75 y 45 años), con una media de escolaridad (E), de 15.6 años (rango entre 12 y 18 años); y 8 mujeres con una media de edad de 61.2 años (rango entre 71 y 44 años), y media de E de 09.13 años (rango entre 16 y seis años). Todos con un diagnóstico de DM2 según registro en el expediente clínico de la institución pública de afiliación. Las características sociodemográficas de los participantes se resumen en la Tabla 2.

El número de años de escolaridad fue el criterio de nominación de los dos grupos de tratamiento previamente formados por su afiliación a la institución pública de salud. Los afiliados a la institución 1 (mayor escolaridad) correspondieron al G1 y los afiliados a la institución 2 (menor escolaridad) correspondieron al G2.

Todas las mediciones del estudio se hicieron en un salón acondicionado con mesas, sillas y equipo audiovisual, 
proporcionado por las instituciones de salud a la que pertenecían los participantes.

\section{Variables}

VI. Años de escolaridad (E).

VD. Calificación en SP y Glucemia.

\section{Instrumentos de evaluación}

Diagnosticados con DM2 y de acuerdo con participar en el estudio, los candidatos de ambas instituciones accedieron a que se les realizaran mediciones de glucosa y en un solo documento (Anexo 1), firmaron un protocolo de consentimiento informado y completaron una cédula con sus datos personales.

Para medir las habilidades de SP, antes y después del taller, los participantes contestaron el Inventario de SP

Tabla 1.

Reactivos del inventario de Solución de Problemas (SP) organizados por factores

\begin{tabular}{|c|c|}
\hline Factores & Reactivos \\
\hline \multirow{4}{*}{$\begin{array}{l}\text { Control } \\
\text { personal }\end{array}$} & $\begin{array}{l}\text { Cuando mis primeros esfuerzos por resolver un problema fallan dudo acerca de mi habilidad para } \\
\text { manejar la situación. }\end{array}$ \\
\hline & Muchos problemas me parecen demasiado complejos como para que los resuelva. \\
\hline & $\begin{array}{l}\text { Algunas veces no reflexiono sobre mis problemas y no trato de resolverlos, sino que tomo una actitud } \\
\text { irreflexiva. }\end{array}$ \\
\hline & $\begin{array}{l}\text { Aunque haya trabajado en un problema algunas veces siento que divago y no llego a la verdadera } \\
\text { esencia de lo que sucede. } \\
\text { Hago juicios precipitados y luego me arrepiento. }\end{array}$ \\
\hline \multirow{5}{*}{$\begin{array}{l}\text { Estilos de } \\
\text { aproximación }\end{array}$} & $\begin{array}{l}\text { Cuando me enfrento con un problema me detengo y pienso acerca de él antes de dar el siguiente } \\
\text { paso. }\end{array}$ \\
\hline & Cuando tomo una decisión evalúo las consecuencias de cada alternativa y las comparo entre sí. \\
\hline & Trato de predecir el resultado final cuando aplico una estrategia de acción. \\
\hline & $\begin{array}{l}\text { Tengo un método sistemático para comparar alternativas y tomar decisiones. } \\
\text { Cuando me enfrento con un problema generalmente no analizo que situaciones extremas puedan } \\
\text { estar contribuyendo al problema. }\end{array}$ \\
\hline & $\begin{array}{l}\text { Cuando estoy confundido por un problema, una de las primeras cosas que pienso es hacer un examen } \\
\text { cuidadoso de la situación y considerar todas las piezas de información relevante. }\end{array}$ \\
\hline \multirow{4}{*}{$\begin{array}{l}\text { Confianza en } \\
\text { la solución } \\
\text { de } \\
\text { problemas }\end{array}$} & Generalmente soy capaz de aportar formas alternativas y creativas para resolver un problema. \\
\hline & $\begin{array}{l}\text { Tengo la habilidad para resolver la mayoría de los problemas, inclusive aquellos que no tienen } \\
\text { solución aparente. }\end{array}$ \\
\hline & $\begin{array}{l}\text { Cuando me enfrento con situaciones nuevas tengo confianza en que puedo manejar los problemas } \\
\text { que surjan. }\end{array}$ \\
\hline & onfío en mi habilidad para resolver nuevos y difíciles problemas. \\
\hline
\end{tabular}

Nota. Clasificación de acuerdo con la adaptación para universitarios mexicanos del instrumento original en inglés de Hepner y Petersen (en Bravo, 1999).
(Bravo, 1999). Este es un instrumento de auto reporte, en versión impresa, con 16 reactivos de cinco opciones de respuesta (Totalmente de acuerdo, De acuerdo, Indeciso, En desacuerdo y Totalmente en desacuerdo); organizados en 3 factores: control personal (6 reactivos), estilos de aproximación (6 reactivos) y confianza en la SP (4 reactivos). La confiabilidad, calculada mediante el alpha de Cronbach, fue de $\alpha=0.83$ para todo el instrumento, los valores alpha para la confiabilidad de las dimensiones de Control personal, Estilos de aproximación y Confianza en la solución de problemas fueron $0.73,0.69$ y 0.70 respectivamente. La clasificación del total de reactivos se muestra en la Tabla 1. 
Tabla 2.

Estadísticos descriptivos de Variables Personales de los grupos G1 y G2.

\begin{tabular}{lcccc}
\hline \multirow{2}{*}{$\mathrm{N}=20$} & \multicolumn{2}{c}{$\mathrm{G} 1$} & \multicolumn{2}{c}{$\mathrm{G} 2$} \\
& $\mathrm{n}=10$ (Hombres) & $\mathrm{n}=10$ (2 Hombres y 8 mujeres) \\
\cline { 2 - 5 } & $\mathrm{Edad}$ & 15.60 & $\mathrm{Edad}$ & $\mathrm{E}$ \\
\hline Media & 57.40 & 2.066 & 63.80 & 10.50 \\
$D E$ & 8.127 & 12 & 9.390 & 4.453 \\
Mínimo & 45 & 18 & 44 & 6 \\
Máximo & 69 & 75 & 16 \\
\hline
\end{tabular}

Nota. Las columnas muestran los datos para grupo1 (G1) y grupo 2 (G2). E refiere a la variable número de años de escolaridad.

Los índices de Glucemia se obtuvieron con un glucómetro One Touch Ultra ${ }^{1}$, para calcular la concentración $(\mathrm{mg} / \mathrm{dl})$ de glucemia capilar.

\section{Diseño y Procedimiento}

Se empleó un diseño ex post facto correlacional prospectivo simple que se caracteriza porque los participantes son seleccionados por una característica que poseen; la Variable Independiente (VI) no se manipula debido a que ya sucedió (Kerlinger, 1979); recibe el nombre de prospectivo cuando se desea identificar el efecto de la VI; y es simple cuando se estudiar una sola VI. A pesar de sus limitaciones, es útil cuando se está haciendo un sondeo hacia algún fenómeno porque permite identificar covariación entre las variables de interés. El tamaño del efecto no se estableció a priori.

Una vez establecido el contacto con dos instituciones públicas de atención a la salud y después de que los directivos conocieron y autorizaron el proyecto, así como la intervención mediante la impartición de un taller en SP conducido por el mismo grupo de psicólogos responsables del proyecto, se programó una entrevista grupal con los adultos diagnosticados con DM2. Antes de solicitarles su firma de consentimiento informado y acordar las indicaciones

\footnotetext{
${ }^{1}$ La glucemia capilar se mide mediante un pinchazo en un dedo para extraer una gota de sangre que luego se coloca en una tira reactiva y se analiza mediante un glucómetro, que mide el contenido de glucosa en la sangre $(\mathrm{mg} / \mathrm{dl})$, exponiendo a la tira reactiva al paso de pequeña corriente eléctrica, cuya respuesta es cuantificada por el medidor. Los resultados están
}

para presentarse a la medición de glucosa y evaluación inicial de las habilidades para solucionar problemas, a los asistentes se les explicó el objetivo y duración del estudio, así como el carácter de su participación. Los interesados acudieron a su institución de salud en condiciones de ayuno la mañana del día previsto; se obtuvieron sus índices de Glucemia, posteriormente ingirieron alimento durante un receso de quince minutos para evitar prolongar el ayuno, firmaron el consentimiento informado, respondieron tanto la cédula de datos personales como el pretest del inventario de SP y recibieron el calendario de las sesiones del taller; inmediatamente después, se inició la primera sesión.

El taller de capacitación tuvo una duración de ocho horas, durante cuatro sesiones semanales de dos horas, el pretest y el postest requirieron dos horas adicionales, una para cada condición. La primera sesión del taller, tuvo como objetivo el que los asistentes conocieran la importancia de la técnica, etapas que la componen y ventajas para el auto manejo de la DM2. En la segunda, los asistentes practicaron la identificación de problemas en situaciones simuladas relacionadas con el auto manejo de la DM2. En la tercera se utilizaron situaciones reales propuestas por los participantes, con el objetivo de ejercitar la generación de alternativas de solución y elección de la más efectiva a partir de efectos, costos, beneficios, recursos y apoyos sociales disponibles. La cuarta y última sesión se dedicó a la

calibrados a plasma con el propósito de comparar fácilmente el resultado con datos de laboratorio. La correlación obtenida al contrastar el dato de mediciones de glucemia capilar con resultados de laboratorio es $r=0.984$ (Jhonson y Jhonson, 2008). 
Tabla 3.

Resultados prueba de Rangos de Wilcoxon para Calificación SP y Glucemia.

\begin{tabular}{|c|c|c|c|c|c|c|c|c|c|c|c|c|}
\hline \multirow{3}{*}{ Grupo } & \multicolumn{6}{|c|}{ Calificación SP } & \multicolumn{6}{|c|}{ Glucemia } \\
\hline & \multicolumn{2}{|c|}{$\mathrm{M}$} & \multicolumn{2}{|c|}{$D E$} & \multirow[b]{2}{*}{ z } & \multirow[b]{2}{*}{$p$} & \multicolumn{2}{|c|}{$\mathrm{M}$} & \multicolumn{2}{|c|}{$D E$} & \multirow{2}{*}{ z } & \multirow{2}{*}{$p$} \\
\hline & Pretest & Postest & Pretest & Postest & & & Pretest & Postest & Pretest & Postest & & \\
\hline $\begin{array}{c}\mathrm{G} 1 \\
\mathrm{n}=10\end{array}$ & 36 & 48 & 7.5 & 8 & $\begin{array}{c}- \\
2.81\end{array}$ & 0.005 & 160 & 128 & 19.0 & 11.0 & $2 . \overline{-}$ & 0.005 \\
\hline $\begin{array}{c}\mathrm{G} 2 \\
\mathrm{n}=10\end{array}$ & 36 & 40 & 7.1 & 6 & $1 . \overline{60}$ & 0.108 & 122 & 170 & 37.2 & 47.2 & 2.31 & 0.021 \\
\hline
\end{tabular}

Nota. La calificación máxima para SP es de 64 puntos; el diagnóstico de diabetes se emite con una concentración plasmática de glucosa en ayuno $\geq 126 \mathrm{mg} / \mathrm{dl}$.

planificación e instrumentación de la alternativa elegida a partir de la jerarquización y establecimiento de metas. Al finalizar las cuatro sesiones de intervención se entregó a los participantes un tríptico con resumen de los objetivos y contenido de las sesiones efectuadas.

Antes y después del taller de capacitación en habilidades de SP, se obtuvieron mediciones de habilidad en SP y Glucemia. Las mediciones se capturaron y analizaron mediante la versión 24 del SPSS. El único dato perdido se calculó como perdido y no hubo mortalidad experimetal.

\section{Resultados}

La Tabla 2 muestra características sociodemográficas de los dos grupos formados. El grupo con mayor escolaridad (E) fue el G1 (número de años escolares promedio $=15.60)$ y el grupo de menor escolaridad fue el G2 (promedio de años en la escuela $=10.50$ ). Ambos grupos presentaron una buena disposición para el cuidado de la salud, en la medida que acudían asiduamente a su institución sanitaria para la supervisión profesional de su glucosa y aceptaron participar voluntariamente en el taller al que fueron invitados.

La prueba U de Mann Whitney (Siegel y Castellan, 1995) aplicada a las mediciones de la variable escolaridad (E) arrojó diferencias significativas entre ambos grupos $(\mathrm{U}=17.000, Z=-2.638, p \leq .05)$.
Las dos variables en las que diferían los grupos antes de iniciar el taller de capacitación en SP, fueron un primer punto de análisis. Se supuso desde un inicio que ambas podrían, sin duda, contribuir significativamente a los resultados que pudieran obtenerse; sin embargo, se decidió tomar como principal variable de análisis a la E que reportaron los participantes, ya que se ha dicho que el nivel de escolaridad define oportunidades y niveles de competencias para el cuidado de la salud (De Vincezi y Tudesco, 2003).

Así, el análisis se orientó hacia la identificación de las diferencias de los resultados de Glucemia y calificación en SP, tanto en el nivel intragrupo como entre grupos, teniendo como variable básica de contraste el grupo al que pertenecían, que se caracterizaba por alta o baja escolari$\operatorname{dad}(\mathrm{E})$.

\section{Análisis Intragrupo}

En la Tabla 3, se muestran los resultados de la prueba de Wilcoxon (Siegel y Castellan, 1995), que exhibió el contraste entre pretest y el postest de los resultados obtenidos dentro de cada grupo (intragrupo). No obstante que ambos grupos presentaron puntajes mayores en la calificación de SP después de participar en el taller, lo que sugiere que todos los participantes incrementaron su habilidad en SP, la diferencia solamente resultó estadísticamente significativa para el G1.

Además, debe notarse que, en el pretest, la media de Glucemia para ambos grupos fue mayor al valor estable- 
Tabla 4.

Resultado del contraste entre G1 y G2 al comparar la diferencia entre condiciones pretest y postest para Glucemia y calificación de SP, con la prueba U de Mann-Whitney para muestras independientes.

\begin{tabular}{lcc}
\hline & Glucemia & SP \\
\hline U de Mann-Whitney & 9.50 & 20.00 \\
W de Wilcoxon & 54.50 & 75.00 \\
$Z$ & -2.90 & -2.27 \\
Sig. asintótica(bilateral) & .004 & .023 \\
Significación exacta [2*(sig. unilateral)] & .002 & .023 \\
Tamaño dl efecto (d de Cohen) & -1.23 & 1.13 \\
$r$ & -0.52 & 0.49 \\
\hline
\end{tabular}

Nota. El nivel de significancia fue: $p<0.05$

cido para diagnosticar DM e, incluso, resultó con un valor promedio superior para el caso del G1.

Sin embargo, solo en el G1 la medición de la Glucemia en la condición del pos-test exhibió un decremento. El G2 mostró, contra lo esperado, un incremento entre el pre y el pos-test. Es decir, mientras que para G1 la Glucemia disminuyó significativamente y en proporción inversa con el incremento de SP; para el G2 incrementó, también significativamente, pero en relación directa con la calificación en SP.

Este resultado lleva a considerar la aceptación de las hipótesis planteadas, pero exclusivamente para uno de los grupos estudiados, el G1. En el caso del G2, aunque exhibió un incremento en el puntaje de SP después la participación en el taller de SP, éste no fue estadísticamente significativo; además de que, respecto a los puntajes de Glucemia, se observó un incremento significativo después de la asistencia al taller de SP, resultado opuesto al esperado.

De acuerdo con lo que se resume en las Tablas 2 y 3 , podemos observar que cuando $\mathrm{E}$ muestra comparativamente un valor más alto, las puntuaciones pre y post de SP exhiben una diferencia significativa; además, se observa también una diferencia significativa entre las puntuaciones pre y pos-test en los índices o mediciones la Glucemia. En contraste, cuando E comparativamente muestra valores inferiores, la diferencia entre puntuaciones pre y post de SP resulta no ser estadísticamente significativa; y tampoco existe diferencia significativa entre las puntuaciones en los pre y pos-test en las mediciones de Glucemia.

Este resultado nos lleva a suponer que los años de escolaridad (la variable E), están asociados a la adquisición o fortalecimiento de habilidades en la solución de problemas; lo que a su vez ha incidido, probablemente, en la disminución significativa de los índices de Glucemia que se observaron entre la condición pretest y postest en el G1.

El resultado del G2, que de entrada mostró menor escolaridad, fortalece el mismo supuesto; en la medida que, aunque recibió por igual la programación del taller conducido por el mismo grupo de psicólogos y en una situación semejante, obtuvo puntajes que no resultaron significativamente diferentes entre las condiciones antes y después del taller de SP y sus índices de Glucemia no disminuyeron.

\section{Análisis entre grupos}

Como una forma de verificar el supuesto de que la variable E probablemente medió el resultado exitoso observado en el G1, se practicó un análisis adicional mediante la comparación entre los pretest y postest de los grupos, con la aplicación de la prueba de U de Mann-Whitney y cuyos resultados se resumen en la Tabla 4.

Se observa que, para ambos grupos, la diferencia de puntajes entre las mediciones del pretest y del postest es significativa para el caso de las mediciones de los índices 
de Glucemia y también en el caso de SP, resultado que fortalece el supuesto de que efectivamente los grupos se comportaron diferente a pesar de haber recibido el mismo tratamiento durante la fase de intervención. El tamaño del efecto calculado muestra que las diferencias entre los grupos mayor $\mathrm{E}(\mathrm{G} 1)$ y menor $\mathrm{E}(\mathrm{G} 2)$ se pueden estimar como grandes, tanto para el caso del control de la Glucemia como para las habilidades para solucionar problema.

El tamaño del efecto permite concluir, sin lugar a duda, que la diferencia puede ser atribuida a que el grupo G1 tuvo un promedio de E significativamente mayor que el G2; y, por lo tanto, E covaría con la adquisición o fortalecimiento de habilidades SP, traduciéndose positivamente en disminución de los índices de Glucemia.

\section{Discusión}

Las hipótesis planteadas como guías de este estudio logran una buena confirmación para el caso de uno de los grupos, el G1, que fue el que contó con un mayor grado de escolaridad. De inicio, este resultado lleva a reformular la relación entre el desarrollo de habilidades en SP, su impacto en el autocuidado de la DM2 y en consecuencia en la mejoría del control de la Glucemia. Por un lado, debemos precisar que no basta poseer un buen conocimiento de las habilidades en SP; sino que hay que poseer las habilidades y destrezas para poner en práctica este conocimiento; que, en nuestro caso, suponemos que a diferencia del G2, el G1 las poseía gracias a su mayor nivel de escolaridad.

Debe hacerse notar que el resultado exitoso para G1 covarió significativamente con la variable $\mathrm{E}$, que se detectó con una media mayor desde la nominación de los grupos. Este resultado concuerda con el trabajo de De Vincezi y Tudesco (2003), quienes reportaron que la educación es condición necesaria en la promoción de salud tanto individual como comunitaria, porque un individuo educado tiene acceso a identificar y utilizar sus derechos y fortalecer sus habilidades para tomar las mejores decisiones en circunstancias que ponen en riesgo su vida.

En resumen, la evidencia expuesta proporciona elementos para presumir que la variable E participó en la me- jor asimilación de los contenidos del taller de SP en el caso del G1 con E mayor; y, en consecuencia, la aplicación de las mismas habilidades en la toma de decisiones saludables para el auto cuidado de la DM2, que se reflejaron en la disminución significativa de la Glucemia del mismo grupo. No así para el grupo G2 con E menor, característica que probablemente determinó un menor efecto de la capacitación y por consiguiente en los índices de Glucemia.

Otro aspecto importante para analizar con mayor profundidad es la diferencia entre las calificaciones de SP de las condiciones pretest y postest, que resultó significativa para G1, pero no para G2; es el hecho de que el instrumento utilizado para la comparación fue elaborado y validado con una muestra de estudiantes universitarios mexicanos, lo que nuevamente dejó en desventaja a los participantes del G2 por razones de escolaridad. Probablemente el lenguaje utilizado o la estructura gramatical de los enunciados fueron mejor comprendidos por el G1, pero no ocurrió igual para el G2, lo que representa una limitante del estudio.

El presente trabajo muestra que, como lo plantea cualquier teoría de diseño instruccional (Reigeluth, 2012), el desarrollo didáctico, la organización de secuencias de aprendizaje y la complejidad de las actividades de un taller como el de SP, deben partir y conducirse con base en el nivel de los conocimientos y habilidades de los participantes. Incluso, si este tipo de factor es pasado por alto en el diseño de las experiencias de aprendizaje que son la base de los talleres SP, el resultado puede llegar a ser contraproducente.

En nuestra experiencia, resulta evidente que, si no se toma en cuenta la escolaridad de los participantes en la programación de un taller de SP, asumiendo que ésta guarda estrecha relación con las habilidades y conocimientos de los participantes, será difícil logar que se alcancen los objetivos de la capacitación en detrimento de los pacientes que se esperan que aprender a vivir con DM2.

De igual forma, si fuera el caso, es conveniente emplear instrumentos de medición validados con grupos normativos diferenciales en edad y escolaridad, para que sean sensibles a las características de los participantes. Resulta 
evidente que el trabajo requiere ser replicado con grupos más grandes y heterogéneos en diversas variables sociales, con el fin de aumentar la validez externa de estos resultados.

En síntesis, el éxito de la capacitación en SP en el autocuidado de la DM2, requiere evaluar exhaustivamente los conocimientos y las habilidades de quienes necesitan integrar un cambio en el estilo de vida, para aprender a vivir con DM2. Consideramos que la escolaridad puede ser un buen indicador del grado en que se poseen estos conocimientos y habilidades.

\section{Agradecimientos}

Este trabajo se realizó con recurso aprobado por la Dirección General de Asuntos del Personal Académico de la Universidad Nacional Autónoma de México, Programa de Apoyo a Proyectos de Investigación e Innovación Tecnológica, IT300114.

\section{Referencias}

American Association of Diabetes Educators. (2018). AADE7 Self-Care Behavior. https://www.diabeteseducator.org/living-withdiabetes/aade7-self-care-behaviors

Arias, J., Villasís, M. y Miranda, M. (2016) The Research Protocol III. Study population. Revista Alergia México, 63(2), 201-206.

Bravo, S. (1999). Comparación en el manejo de habilidades para solucionar problemas sociales en sujetos con diferente predisposición al alcoholismo. [Tesis de Licenciatura]. Universidad Nacional Autónoma de México, México.

Chan, M. (2016). Obesidad y diabetes, una plaga lenta pero devastadora: discurso inaugural de la Directora General de la OMS en la $47^{a}$ reunión de la Academia Nacional de Medicina. Recuperado de http://www.who.int/dg/speeches/2016/obesitydiabetes-disaster/es/
De Vincezi, A. y Tudesco, F. (2003). La educación como proceso de mejoramiento de la calidad de vida de los individuos y en la comunidad. Revista Iberoamericana de Educación, 49(7), 1-12.

D'Zurilla, T. J. y Nezu, A. M. (2010). Problem-Solving Therapy. En K. Dovson (Ed.), Handbook of Cognitive-Behavioral Therapies $\left(3^{\circ}\right.$ ed.; pp. 197225). The Guilford Press.

D'Zurilla, T. J. y Goldfried M. R. (1971). Problem Solving and Behavior Modification. Journal of Abnormal Psychology, 78, 107-126. https://doi.org/10.1037/h0031360

García, R. y Sánchez, J. J. (2013). Efectos de la entrevista motivacional en el tratamiento de la diabetes mellitus tipo 2 [Effects of the Motivacional Interview in the Treatment of Diabetes Mellitus Type 2]. Psicología y Salud, 23(2), https://doi.org/10.25009/pys.v23i2.500

183-193.

Hill-Briggs, F. (2003). Problem Solving in Diabetes selfManagement: A Model of Chronic Illness SelfManagement Behavior. Annals of Behavioral Medicine, 25(3), 182-193. https://doi.org/10.1207/S15324796ABM2503_04

Hill-Briggs F. y Gemmell L. (2007). Problem Solving in Diabetes Self-Management And Control: A Systematic Review of the Literature. Diabetes Educator, 33(6), 1032-1050. https://doi.org/10.1177/0145721707308412

Instituto Nacional de Salud Pública de México. (2018). Encuesta nacional de salud y nutrición 2018. Presentación de resultados. Secretaría de Salud, Instituto Nacional de Salud Pública e Instituto Nacional de Estadística, Geografía e Informática. Recuperado de https://ensanut.insp.mx/encuestas/ensanut2018/do ctos/informes/ensanut 2018 presentacion resulta dos.pdf

Interial, Ma. G., Campillo, C. y Aguilar, I. (2013). Análisis comparativo del programa de educación en 
diabetes mellitus de México y Cuba [Comparative Analysis of the Education Program in Diabetes Mellitus in Mexico and Cuba]. Revista Cuidarte. Programa de enfermería UDES, 4(1) 516-522. https://doi.org/10.15649/cuidarte.v4i1.12

Jhonson \& Jhonson Medical. (2008). One Touch. Manual del Usuario. Sistema de monitoreo de glucosa en la sangre. Jhonson \& Jhonson.

Kerlinger, F. (1979). Enfoque conceptual de la investigación del comportamiento. Interamericana.

Lazcano, M. y Salazar, B. (2007). Estrés percibido y adaptación en pacientes con diabetes mellitus tipo 2 [Perceived Stress and Adaptation in Patients with Type 2 Diabetes mellitus]. Aquichan, 7(1), 77-84.

Nezu, C. M., Nezu, A. M. y Colosimo, M. M. (2015). Case Formulation and the Therapeutic Alliance in Contemporary Problem-Solving Therapy (PST). Journal of Clinical Psychology: In session, 71(5), 428-438. https://doi.org/10.1002/jclp.22179

Organización de las Naciones Unidas para la Educación la Ciencia y la Cultura. (2020). Educación para la salud $y$ el bienestar. Recuperado de https://es.unesco.org/themes/educacion-salud-y$\underline{\text { bienestar }}$

Organización Mundial de la Salud. (2020a). Diabetes. Recuperado de https://www.who.int/es/newsroom/fact-sheets/detail/diabetes

Organización Mundial de la Salud. (2020b). Perfiles de los países para la diabetes. Recuperado de https://www.who.int/diabetes/countryprofiles/mex es.pdf?ua $=1$

Powers, M. A., Bardsley, J., Cypress, M., Duker, P., Funnell, M. M., Fischl, A. H., Maryniuk, M. D., Siminerio, L. y Vivian, E. (2017). Diabetes SelfManagement Education and Support in Type 2
Diabetes: A Joint Position Statement of the American Diabetes Association, the American Association of Diabetes Educators, and the Academy of Nutrition and Dietetics. The Diabetes Educator, 43(1) 41-53. https://doi.org/org/10.1177/0145721716689694

Reigeluth, C. (2012). Teoría instruccional y tecnología para el nuevo paradigma de la educación [Instructional Theory and Technology for the New Paradigm of Education]. Revista de Educación a Distancia, $\quad 32, \quad 1-22$. https://doi.org/10.6018/red/50/1a

Riveros, A., Cortazar, J., Alcazar, F. y Sánchez, J.J. (2005). Efectos de una intervención cognitivoconductual en la calidad de vida, ansiedad, depresión y condición médica de pacientes diabéticos e hipertensos esenciales. International Journal of Clinical and Health Psychology, 5(3), 445-462.

Siegel, S. y Castellan, N. J. (1995). Estadística no paramétrica, aplicada a las ciencias de la conducta. Trillas.

Schumann, K. P., Sutherland, J. A., Majid, H. M., Haseeb, M. y Hill-Briggs, F. (2011). Evidencebased Behavioral Treatments for Diabetes: Problem-solving therapy. Diabetes Spectrum, 24(2), 64-69. https://doi.org/10.2337/diaspect.24.2.64

Sotaminga, M. (2019). Diseño instruccional en sector Salud. Recuperado de https://www.researchgate.net/publication/3319840 37 Diseno instruccional en sector Salud

Zhang J, Marmor, R., y Huh, J. (2018). Towards Supporting Patient Decision-making in Online Diabetes Communities. AMIA, Annual Symposium Proceedings, 2017, 1893-1902. 


\section{Anexo 1 \\ UNIVERSIDAD NACIONAL AUTÓNOMA DE MÉXICO \\ FACULTAD DE ESTUDIOS SUPERIORES IZTACALA \\ CARRERA DE PSICOLOGÍA}

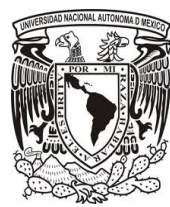

El propósito de esta carta es formalizar el consentimiento informado de los usuarios, después de informarles de la investigación titulada IMPACTO DE CUATRO TRATAMIENTOS SOBRE EL COMPORTAMIENTO DE ADHERENCIA A LA DIETA EN PACIENTES

DIABÉTICOS, así como el papel que desempeñarán.

El estudio es conducido por psicólogos adscritos a la Facultad de Estudios Superiores Iztacala, de la UNAM. El objetivo es que los usuarios diagnosticados con Diabetes Mellitus 2, mejoren la adherencia a la dieta prescrita por su médico tratante, a través de un taller. La participación en este estudio es estrictamente voluntaria, la información que se obtenga será confidencial y los datos se utilizarán con fines de análisis y publicación, siempre resguardando los datos personales de los participantes. Algunas sesiones de trabajo se video-grabarán con fines didácticos.

Si tiene duda sobre el presente estudio, puede aclararla en el momento que usted desee. Igualmente, puede retirase del proyecto cuando usted lo decida, sin que eso le perjudique de ninguna forma.

Agradecemos su participación.

Sirva la presente para manifestar que por voluntad propia doy mi consentimiento para recibir atención psicológica de parte del proyecto IMPACTO DE CUATRO TRATAMIENTOS SOBRE EL COMPORTAMIENTO DE ADHERENCIA A LA DIETA EN PACIENTES DIABÉTICOS, que es conducido por psicólogos adscritos a la Facultad de Estudios Superiores Iztacala, de la UNAM. Declaro que recibí una explicación clara y completa de la naturaleza del tratamiento, motivo por el cual decidí aceptar. Entiendo que puedo dar por terminada mi participación en el taller en cualquier momento sin recibir sanción alguna. También recibí información de la forma en que se utilizarán los datos, entiendo que se publicarán trabajos en revistas de difusión científica, siempre conservando mi anonimato y no se comunicarán a nadie más sin mi consentimiento.

Tlalnepantla, Estado de México, a de del 20 . 
NOMBRE Y FIRMA DEL PARTICIPANTE

TALLER PAR MEJORAR LA ADHERENCIA A LA DIETA EN PACIENTES CON DIABETES MELLITUS 2

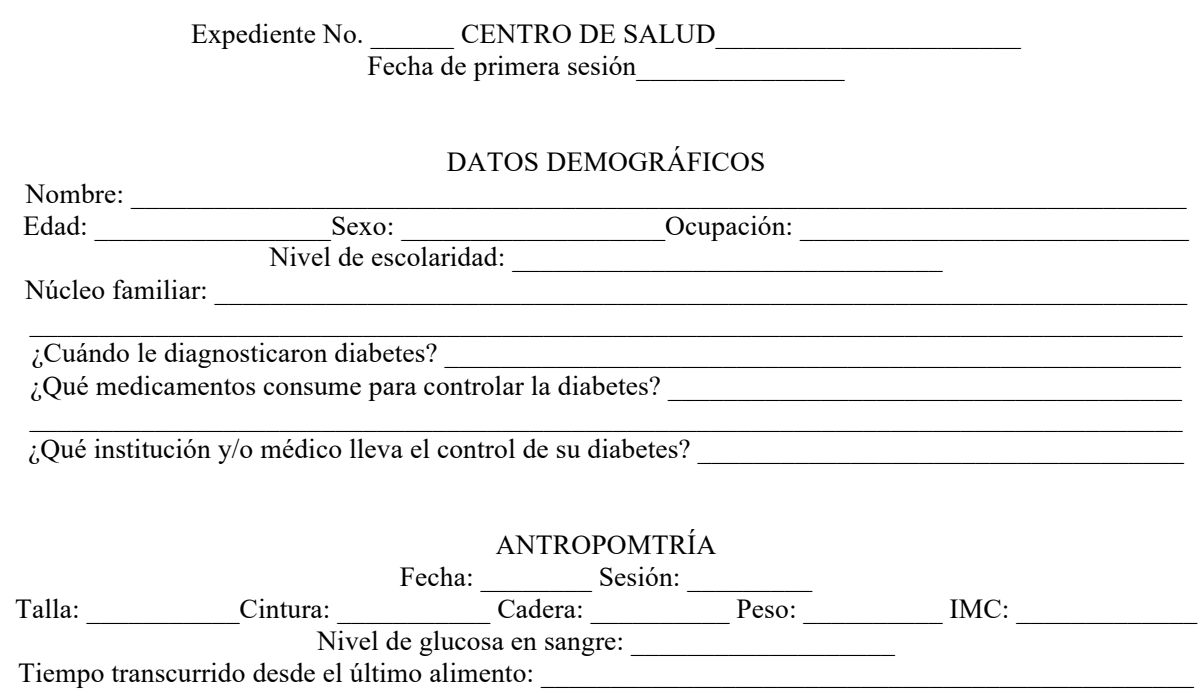


